\title{
Proceedings of the $\mathrm{XX}$ congress of the Italian Society for Pure and Applied Biophysics (SIBPA) Arcidosso (Gr), Italy, September 2010
}

\author{
Daniela Giacomazza • Franco Gambale
}

Received: 14 October 2011/ Accepted: 15 October 2011

(C) European Biophysical Societies' Association 2011

This issue of the European Biophysics Journal contains seven articles based on lectures presented at the XX Meeting of the Italian Society for Pure and Applied Biophysics (Società Italiana di Biofisica Pura e Applicata, SIBPA), held in Arcidosso (Grosseto, Italy) from 11th to 14th September 2010. The XX meeting of the SIBPA marked a special anniversary for Italian biophysics, as Franco Gambale explained to the audience.

The young Italian biophysicists in the audience learnt that use of the term "biophysics" started in the nineteensixties within physics Institutes. As a matter of fact, the basic question in biophysics had already been introduced in 1944 by the winner of the Nobel prize for physics Erwin Schrödinger in his pioneering book "What is life?". By 1950 , as many as 200 research centers all around the world had already adopted the word biophysics in their name and had started to investigate living matter to answer Schrödinger's question "How can the events in space and time which take place within the spatial boundary of a living organism be accounted for by physics and chemistry?".

Biophysics research in Italy started playing a role mainly because of the forward-looking activity of the Italian scientists Antonino Borsellino, Adriano Gozzini, and Mario Ageno, physics professors at Genoa, Pisa, and Rome Universities, respectively. Indeed, as early as 1959, Professor Antonino Borsellino organized a series of

D. Giacomazza $(\bowtie)$

Consiglio Nazionale delle Ricerche, Istituto di Biofisica

(Palermo Research Division), Via Ugo La Malfa,

153-90146 Palermo, Italy

e-mail: daniela.giacomazza@pa.ibf.cnr.it

F. Gambale

Consiglio Nazionale delle Ricerche, Istituto di Biofisica,

Via De Marini, 6-16149 Genoa, Italy
"Conferences on Biophysics and Biochemistry" in Genoa and, in 1963, the first Italian National Congress of Biophysics in Rome. In 1960 Borsellino also promoted the creation of the Italian Society for Pure and Applied Biophysics, but, unfortunately, owing to a series of impediments, the Society was finally established only in 1973 with Borsellino as its first President.

Borsellino also promoted the participation of Italian biophysicists in the "first" International Congress on Biophysics held in Stockholm, Sweden, from July 31st to August 4th 1961, on the initiative of the International Union of Experimental and Theoretical Physics. This was the first meeting gathering biophysicists from around the world. The International Union of Pure and Applied Biophysics was formed and it decided to convene congresses once every three years. Today, therefore, it seems very appropriate to celebrate in this issue the XX SIBPA Congress and 50 years from the first International Biophysics meeting. The Stockholm agenda included: (1) mathematical modeling of biophysical phenomena; (2) new methods in biophysics (laser technology and others); (3) membrane biophysics and (4) vital problems of the molecular bases of virus replication.

In some respects, these topics, in an updated version, were still part of the debate within the XX SIBPA meeting dealing with: (1) theories, models, and computational methods in biophysics, (2) spectroscopy and microscopy for biophysics and medical physics, and (3) molecular, cellular, and sensory biophysics. The article presented by Gerardo Abbandonato et al. reports the interesting cistrans photoisomerization of two butenolide and two pyrrolinone derivatives sharing close structural relationships with the auto-fluorescent protein (AFP) family. The photoinduced alterations of the physicochemical properties of organic molecules are increasingly being investigated with 
a view to engineering new photoresponsive nanodevices for nanoscale medical applications. These new photochromic molecular systems have remarkably pure optical switching control.

Patch-clamp recording, developed in the late 1970s to directly observe ion channel currents, has rapidly become the most important experimental approach for precise measurement of the electrical properties of plasma membranes. The article by Mascia Benedusi et al. reports a new method of fabrication of patching pipettes, using just one glass type that enables the sealing of a wide variety of cell types isolated from different amphibian, reptilian, fish, and mammalian tissues, and artificial membranes made of different lipid mixtures.

Strength and robustness are properties of fundamental importance to biological materials, and are crucial to providing functional properties for living systems and for the synthetic and biomimetic materials inspired by these. These important features are achieved through complex self-organizing structures formed by ordered assembly of monomeric species. In the article by Francesco Federiconi et al. high-pressure synchrotron X-ray diffraction was used to analyze the mechanical properties of quadruplexes obtained by hierarchical self-assembly of alkaline folates, hence to investigate their strength and robustness.

The advent of nanotechnology has had a major effect on a wide range of medical and biological applications, and on electronics, water-treatment technology, and solar cells. However, little is known about how such systems can modify the structure of the proteins and interfere with their biological activity. Because biological effects at the cellular level depend on molecular interactions, studying the interaction of nanoparticles with biomolecules may be fundamental to understanding uptake and toxicity mechanisms in living organisms. By using a model system, Edi Gabellieri et al. have shown that nanoparticles can interact with proteins thus forming complexes in which the protein structure may be significantly modified.

The interesting review by Albrecht Haase et al. discusses results from experiments conducted to trace asymmetry at different stages along the honeybee's (Apis mellifera) olfactory pathway. Their data were obtained by scanning electron microscopy, electroantennography, and multi-photon microscopy. Finally, possible links to the results of behavioral tests are presented.

The research work by Deleana Pozzi et al. describes the structural and functional effects of exposing murine fibroblasts to therapeutic ultrasound at $1 \mathrm{MHz}$ frequency. They found that the ultrasound induces a statistically significant increase in the concentration of free radicals. These molecular species are known to modify the cellular equilibrium inducing stress effects in the cellular organelles, with dramatic consequences for cell survival.

In recent years metal divalent cations, especially those of copper and zinc, have been investigated for their involvement in the aggregation of the well-known prion protein responsible for the onset of Creutzfeldt-Jakob disease in humans. The article presented by Francesco Stellato et al. discusses SAX measurements on various portions of Prion-protein tetra-octa-repeat peptides in complexes with $\mathrm{Cu}$ (II) ions, both in the presence and absence of $\mathrm{Zn}$ (II). Results show that $\mathrm{Zn}$ acts by directly interacting with the peptide, in this way competing with $\mathrm{Cu}$ for binding with histidine. This finding suggests that metal binding competition can be important in the more general comprehension of metal homeostasis.

Many young scientists had the opportunity to attend the XX SIBPA meeting, because of support provided by the Institute of Biophysics of the Italian National Research Council. We would like to thank all speakers and meeting participants for their valuable contributions to the success of the meeting. Sponsorship by the Comune di Arcidosso, Nikon, Leica Microsystems, Monte dei Paschi di Siena, Crisel Instruments, Corsini, Institute of Cybernetics of the Italian National Research Council, and TA Instruments is also acknowledged. We also express our gratitude to the administrative and technical teams of the Institute of Biophysics of the Italian National Research Council (Pisa Research Division) for their valuable help.

Finally, we would particularly like to thank Anthony Watts and the editorial staff of EBJ for dedicating, for the third time, an issue of this journal to Italian biophysics. 